\title{
An innovate strategy to treat large metaphyseal segmental femoral bone defect using customized design and 3D printed micro-porous prosthesis: a prospective clinical study
}

\section{Guojin Hou}

1.Department of Orthopaedic Surgery, Peking University Third Hospital 2.Engineering Research Center of Bone and Joint Precision Medicine, Ministry of Education, Peking University Third Hospital

\section{Bingchuan Liu}

1.Department of Orthopaedic Surgery, Peking University Third Hospital 2.Engineering Research Center of Bone and Joint Precision Medicine, Ministry of Education, Peking University Third Hospital

\section{Yun Tian ( $\boldsymbol{D}$ tiany@bjmu.edu.cn )}

1.Department of Orthopaedic Surgery, Peking University Third Hospital 2.Engineering Research Center of Bone and Joint Precision Medicine, Ministry of Education, Peking University Third Hospital https://orcid.org/0000-0003-2310-6054

\section{Zhongjun Liu}

1.Department of Orthopaedic Surgery, Peking University Third Hospital 2.Engineering Research Center of Bone and Joint Precision Medicine, Ministry of Education, Peking University Third Hospital

\section{Fang Zhou}

1.Department of Orthopaedic Surgery, Peking University Third Hospital 2.Engineering Research Center of Bone and Joint Precision Medicine, Ministry of Education, Peking University Third Hospital

Hongquan Ji

1.Department of Orthopaedic Surgery, Peking University Third Hospital 2.Engineering Research Center of Bone and Joint Precision Medicine, Ministry of Education, Peking University Third Hospital

\section{Zhishan Zhang}

1.Department of Orthopaedic Surgery, Peking University Third Hospital 2.Engineering Research Center of Bone and Joint Precision Medicine, Ministry of Education, Peking University Third Hospital

\section{Yan Guo}

1.Department of Orthopaedic Surgery, Peking University Third Hospital 2. Engineering Research Center of Bone and Joint Precision Medicine, Ministry of Education, Peking University Third Hospital

\section{Yang Lv}

1.Department of Orthopaedic Surgery, Peking University Third Hospital 2.Engineering Research Center of Bone and Joint Precision Medicine, Ministry of Education, Peking University Third Hospital

\section{Zhongwei Yang}


1.Department of Orthopaedic Surgery, Peking University Third Hospital 2.Engineering Research Center of Bone and Joint Precision Medicine, Ministry of Education, Peking University Third Hospital

\section{Research}

Keywords: virtual surgery, customized design, 3D printed, micro-porous prosthesis, membrane induction, irregular-shaped, segmental femoral bone defect.

Posted Date: February 24th, 2020

DOI: https://doi.org/10.21203/rs.2.24245/v1

License: (c) (i) This work is licensed under a Creative Commons Attribution 4.0 International License. Read Full License 


\section{Abstract}

Background Large segmental bone defect at the metaphyseal area is still difficult to treat, nowadays, there is a tremendous level of interest in uses of 3D printing technology in orthopaedic surgery. This study was introduced to prospectively confirm the safety and effectiveness of 3D printed micro-porous prosthesis in clinical bone defect reconstruction application.

Methods Patients with segmental irregular-shaped bone defect of the femur were recruited from 2017.12 to 2018.11. The first stage of the treatment involves radical debridement of all infected or non-viable bone and interposed fibrous tissue, and temporary fixation. Once the culture and biopsy results were negative, the PMMA spacer should remain in the defect approximately 6-8 weeks. This period is for the membrane formation, virtual surgery (computed tomography (CT) scan of the lesion area and the contralateral parts of the femur, and then design of the implant). The second stage involves reconstruction the defects with the 3D printed micro-porous prosthesis combined with intra-medullar nailwithout bone graft.Routine clinical follow-up and radiographic evaluation were done to assess bone incorporation and complications of internal fixation. The weight-bearing time and the joint function were recorded.

Result 5 consecutive patients were included in the study. They were followed up for an average of 16.4 months. The average length of bone defect and the distal residual bone was $12 \mathrm{~cm}$ and $6.5 \mathrm{~cm}$. The average time of partial weight-bearing and full weight-bearing was 12.7 days and 2.6 months. X-ray demonstrated good osseous integration of the implant/bone interface. No complications occurred such as implant loosening, subsidence, loss of correctionand infection. At the last follow-up, Harris score of hip joint was excellent in 2 cases, good in 2 cases, fair in 1 case; HSS score of knee joint was good in 4 cases, middle in 1 case.

Conclusion Meticulous customized design 3D printed micro-porous prosthesis combined with intramedullary fixation may be a cost-effective and an alternative strategy to treat metaphyseal segmental irregular-shaped femoral bone defect without bone graft, especially for cases with massive juxta-articular bone loss.

\section{Background}

Trauma and infection are the main causes of clinical bone defect [1], large segmental bone defect of limb extremities is difficult to treat because of its large span, different shape and serious damage to local mechanical stability [2, 3], especially at the metaphyseal area. There are many methods to treat bone defect of limb extremities, such as bone transport through distraction osteogenesis, Masquelet membrane induction, autogenous bone transplantation and artificial bone transplantation, but all these traditional methods have shortcomings such as long treatment cycle, unable to bear weight early, high medical cost and even unsatisfactory treatment effect.

Attias et al [4] reconstructed a long segmental defect with a cylindrical titanium mesh cage packed with bone graft and stabilized with limited contact dynamic compression plates. This metal inner support 
improved immediate stability, however it still needed massive bone graft. Nowadays, there is a tremendous level of interest in uses of 3D printing technology in orthopaedic surgery $[5,6]$, especially in the realm of adult reconstructive surgery [7] and joint arthroplasty [8]. And it is rapidly permeating every aspect of orthopaedic surgery, such as trauma [9], spine [10], hand [11] and tumor [12]. These implants are custom designed by 3D printing technology with the cooperation of orthopaedic surgeons, orthopaedic engineers and biomedical engineers. The possibility of patient custom 3D printed implant has opened new alternative for complex post traumatic limb reconstruction. Such novel strategy may also be used to address the extremely difficult problem of segmental bone defect [13]. However, a composite of cancellous allograft and demineralized bone matrix putty may still be needed in most of the literature [14]. Xu and Liu et al [15] reported that application of 3D printing prosthesis in the treatment of defects caused by spinal tumors has achieved good results. These printed prostheses have achieved good bone ingrowth without any bone graft. Our animal experiments [16] also showed that good bone ingrowth around the prosthesis without any bone graft. So, we have innovatively improved this technique and assumed the 3D printing microporous titanium prosthesis combined membrane induction and intramedullary fixation could successfully treat large bone defect without bone graft.

This study was introduced to prospectively confirm the safety and effectiveness of 3D printed microporous prosthesis in clinical bone defect reconstruction application at the metaphyseal part of femur. This study is support by Beijing Municipal Science \& Technology Commission. Several patients of distal femoral irregular-shaped bone defect have been treated successfully. And our cases showed that biomechanical and biological induction can stimulate local osteogenesis at the prosthetic-bone interface without bone grafting. We will address virtual surgical planning and implant design considerations, indicating how this strategy could be successfully introduced into clinical practice.

\section{Methods}

\subsection{Patients}

This was a prospective clinical study conducted at our hospital between 2017.12 and 2018.11. In this study, patients were recruited for 3D printed microporous titanium prosthesis combined with intramedullary fixation without bone graft based on the indications and contraindications as follows according to our limited experience, and few other reports in the literature [17].

Inclusion criteria

1. Femoral bone defect caused by trauma or infection;

2. The length of the bone defect was $>8 \mathrm{~cm}$, particularly when the remaining distal bone is less in length;

3. Patients aging from 18 to 70 years old.

Exclusion criteria 
1. Bone defect caused by tumors;

2. Patients with articular surface defect;

3. Allergy to prosthetic components materials;

4. Systemic diseases contraindicating the use of the artificial prosthesis;

5. Patients unwilling to undergo this strategy or unable to finish the follow-up.

This study was approval by our hospital's Human Research Ethical Committees and the principles outlined in the Declaration of Helsinki were followed in the study. 5 patients with large irregular-shaped metaphyseal bone defect of femoral were recruited. All patients were informed preoperatively regarding the surgical purpose, management protocol, possible complications and recovery period. And informed consent was obtained from all participants. This study was based on data gathered from the hospital electronic medical record (EMR) system.

\subsection{First-stage Surgical procedure}

The patients had undergone radiography of the leg pre- and post-operatively, a radionuclide scan is required if infection is suspected. The first stage involves radical debridement of all infected or non-viable bone and interposed fibrous tissue. The proximal bone/implant interface was cut smoothly, while deal with the distal bone/implant interface, we preserve the non-infected living bone as much as possible, not caring the contour profile. The femur is stabilized with external fixation, or if the defection is noninfectious, we would preserve the original internal fixation. The antibiotic cement spacer is used to fill the voids and the spacer should be configured to mimic the size and shape of the original bone that is to be reconstructed, following the principle of "replace like with like". The temporary construct must be rigid enough to facilitate early mobilization. Adequate soft-tissue coverage could ensure wound healing. Vacuum wound drainage can be used to cover the wound in the early stage of debridement. Once the culture and biopsy results were negative, the wound was deemed clean and ready for reconstruction. The PMMA spacer should remain in the defect approximately 6-8 weeks. This intervening period is for the membrane formation, virtual surgery and planning for secondary reconstruction.

\subsection{Implant design}

Each patient had consented for use of a custom-designed, patient specific 3D-printed titanium implant by Shandong Weigao Orthopaedic Device Company Limited. combined with additional internal fixation. The patients were sent for a computed tomography (CT) scan of the lesion area and the contra-lateral parts of the femur for preoperative planning after debridement. Once the CT images of the patient's irregularshaped bone defect anatomy were available, the manufacturer initiated the design team through the medical-worker interaction platform. The surgeon is invited to provide recommendations for fixation desires. Each product is particular for its personalized design.

The design of the implant must take into consideration a number of critical factors include mechanical, anatomical and functional aspects that are unique to each case to allow the composite construct to successfully incorporate. The prosthesis adopts normal dodecahedron element micro-pore design, with 
porosity of about $70 \%$, pore size of $(625 \pm 70) \mu \mathrm{m}$, elastic modulus of $(1200 \pm 48) \mathrm{MPa}$ and compressive strength of $(66 \pm 0.5) \mathrm{MPa}$. The micro-porous designed interface may provide not only torsional resistance, but also limits the potential for shear across the bone/implant interface. We usually use intramedullary (IM) nail to finish definitive fixation of the bone/implant composite, as it provides immediate stability. IM nails are highly constraining devices and require the bones to be positioned and aligned correctly during both the virtual and the actual procedure. So, the prosthesis is hollow to mimic the medullary cavity and its diameter is $1.0-1.5 \mathrm{~mm}$ larger than that of intramedullary nail for easy insertion of the nail, for our design is a fixation model of prosthesis combined with anterograde or retrograde intramedullary nail. The shape of the prosthesis must respect the original anatomy and configuration of bone. For long bone defects, juxta-articular sides have an irregular-shaped metaphyseal side, and diaphyseal sides are more cylindrical. Doing so limits the potential for soft tissue impingement, retain more living bone, especially the supracondylar part of the femur. Also, the size of prosthesis should be just equal or smaller than that of residual proximal and distal bones, which do not affect bone tissue crawling. If the distal end of the prosthesis is too close to the knee joint or the distal residual bone quality is poor, it is necessary to print a lateral plate, which is $3.5-4 \mathrm{~mm}$ thick, and is connected with the prosthesis reinforcement to ensure strength, and $6.5 \mathrm{~mm}$ cancellous bone screw should be used for fixation, the design of screw hole should avoids interference with intramedullary nail locking screw. Furthermore, the 3-D configuration of the implant must be designed to allow it to be successfully implanted through the planned surgical exposure.

Once a design has been reached, a schematic is sent to the surgeon with hardware projections and dimensions. Supplemental hardware such as trial-sizing implants, trial implant replica, and directional guides are also designed at this time. The implant is manufactured after the surgeon has approved the implant. The prosthesis is printed by electron beam melting augmentation manufacturing equipment, and then support dispel, local polishing, clean, package, and then deliveded to the healthcare institution for inspection by the surgeon. Finally, the implant is sterilized before implantation.

\subsection{Second-stage Surgical procedure}

The second stage follows an interval of approximately 6-8 weeks, to allow the membrane to adequately develop. Of course, this will depend on the soft tissue, the physiologic quality of the patient. At this point the defect site is ready for definitive reconstruction with a custom mircro-porous implant placed into the induced membrane. The reconstruction must include intramedullar nail. The second stage is followed by physiotherapy including immediate unrestricted ROM exercises, and progression to full weight bearing (FWB) over 4-12 weeks.

\subsection{Observation index}

Routine clinical review is conducted at intervals with plain radiographs to assess graft incorporation and to confirm restoration of skeletal continuity. The weight-bearing time of the patients was recorded, and the corresponding scoring system was used to evaluate the joint function after operation. 


\section{Results}

All 5 patients were treated from 2017.12 to 2018.11 . The patient demographic data are presented in the Table 1.

Table 1 General characteristics of the 5 patients

\begin{tabular}{|cccccccccc|}
\hline No. & Sex & Age(y) & Reason & $\begin{array}{l}\text { L(defect) } \\
(\mathrm{cm})\end{array}$ & $\begin{array}{l}\mathrm{L}(\mathrm{DRB}) \\
(\mathrm{cm})\end{array}$ & $\begin{array}{l}\mathrm{T} \\
(\mathrm{PWB}) \\
(\mathrm{d})\end{array}$ & $\begin{array}{l}\mathrm{T} \\
(\mathrm{FWB}) \\
(\mathrm{M})\end{array}$ & $\begin{array}{l}\text { Harris } \\
\text { score }\end{array}$ & $\begin{array}{l}\text { HSS } \\
\text { score }\end{array}$ \\
\hline 1 & $\mathrm{M}$ & 27 & Infection & 12 & 12 & 4 & 3 & 89 & 65 \\
\hline 2 & $\mathrm{~F}$ & 42 & Trauma & 15.5 & 3.7 & 14 & 4 & 96 & 83 \\
\hline 3 & $\mathrm{~F}$ & 64 & Infection & 12.5 & 4.3 & 14 & 3 & 90 & 82 \\
\hline 4 & M & 61 & Infection & 11.5 & 4.7 & 21 & 3 & 89 & 75 \\
\hline 5 & F & 69 & Infection & 8.5 & 8 & 30 & 2 & 71 & 70 \\
\hline
\end{tabular}

(L: length; T: time; DRB: distal residual bone; PWB: partial weight bearing; FWB: total weight bearing)

The average age of the 5 patients was 52.6 years. There were 2 males and 3 females. 5 patients were followed up for an average of 16.4 months. The causes of bone defect were infection in 4 cases (Figure 1) and trauma in 1 case (Figure 2). The length of bone defect was 8.5-15.5 cm (average $12 \mathrm{~cm}$ ), the length of distal residual bone was $3.7-12 \mathrm{~cm}$ (average $6.5 \mathrm{~cm}$ ). The average time of partial weight-bearing was 12.7 days. The average time of full weight-bearing was 2.6 months. All patients had good stability after operation. No complications such as looseness and infection of internal fixation occurred. At the last follow-up, Harris score of hip joint was excellent in 2 cases, good in 2 cases, fair in 1 case, excellent and good rate was $80 \%$; HSS score of knee joint was good in 4 cases, middle in 1 case, good rate was $80 \%$.

The 12-month postoperative X-ray demonstrated good osseous integration of the implant and the bone nearby. From the AP and lateral position of X-ray, we can observe that the callus crawls out from the residual bone to the peri-prosthesis, wraps around the prosthesis, and forms a stable structure between the prosthesis and bone, which is similar to the bone healing in a relatively stability. No early signs of subsidence, implant collapse, or loss of correction were seen at a mean follow-up point of 16.4 months.

\section{Discussion}

Segmental bone loss further complicates attempts at reconstruction after severe initial injury, it is often arduous for both surgeon and patient [18]. The distal metaphyseal part of femur is a cylindrical-like structure with gradually enlarged distal diameter and irregular shape. Segmental bone loss at this area is difficult to handle for the difficulty in reconstruction to its normal irregular shape, large demand of bone graft to achieve union and poor stability to allow weight bearing at the early stage, especially when there 
is less bone residual near the articular surface. Traditional methods such as bone lengthening, Masquelet membrane induction and autogenous bone graft, may need a long treatment period and unable to bear weight at early time. In recent years, the application of 3D printing technology in patient-specific orthopedics has developed rapidly. Introducing 3D printing technology into the treatment of irregular bone defect could rapidly realize anatomical reconstruction of bone defect, achieve mechanical conduction similar to original bone and promote bone/prosthesis osteogenesis, finally achieve relative stability fusion of bone/prosthesis interface. There are some key points of this innovative method to successfully treat segmental bone defect.

\subsection{Reconstruction of mechanical stability and force conduction}

We use the following methods to obtain the local early mechanical stability. 1) Choose the mechanical conduction mode of the combination of intramedullary nail and prosthesis. The length of the intramedullary nail goes forward and reaches the distal end of the femoral condyle. If retrograde nail was selected, it reaches the less trochanter. 2) The long axis of the prosthesis refers to the curvature of the femur and matches the curvature radius of the intramedullary nail, the prosthesis can only slide along the long axis, without any rotation and angulation. The conduction of the concessional force is matched with the direction of the femoral long axis, and the stress can be better dispersed. 3) The proximal end of the prosthesis is in contact with the smooth femoral stump, the distal end of the prosthesis matches with the irregular bone stump, which ensures that the strength transmitted from the proximal part is evenly transmitted to the distal part. 4) The mechanical properties of the prosthesis can simulate normal bone tissue (elastic modulus of ( $1200 \pm 48) \mathrm{MPa}$ and compressive strength of $(66 \pm 0.5) \mathrm{MPa})$. 5) A lateral plate may be added at the distal end of the prosthesis according to the residual bone of the femoral condyle and can be fixed with screw to increase the stability of the prosthesis. Our structural design experience is shown in Fig. 3. All of the above factors may facilitate early weight-bearing and functional exercise, and help patients recover social function as soon as possible. Our case demonstrated a relative short weight-bearing time, which is 12.7 days for partial weight-bearing and 2.6 months for full weightbearing, with good joints function nearby.

\subsection{Factors promoting the osteogenesis of the defection region}

Fracture healing depends on local mechanical stability and good biological environment [19], but for our study it is prosthesis-osseo-integration. Our previous experiments [20] have proved that bone tissue can grow well into the pore of 3D printed metal prosthesis without bone graft. There are several factors that contribute to the prosthesis-osseo-integration in our study: 1) Appropriate pore size $((625 \pm 70) \mu \mathrm{m})$ is conducive to the in-growth of bone tissue, and the elastic modulus of 3D-printed micro-porous prosthesis can be adjusted by porosity to make it close to the target area. 2) Reduce the size of prosthesis compared 
with the original configuration of bone, especially in the proximal part, which may not affect bone tissue crawling, facilitate interface fusion and reduce the potential for soft tissue impingement. 3) Preclinical studies [21] have shown that induced membranes are highly vascularized and rich in growth factors. Furthermore, they have osteogenic and osteoinductive properties, and associated with the expression of various key genes related to angiogenesis and osteogenesis [22]. 4) Prosthesis' unidirectional fretting along the long axis of femur because of the matched curvature radius of the prosthesis and intramedullary nail, the prosthesis can only slide along the nail, this would stimulate osteogenesis at the bone end. 5) Because the prosthesis and bone are not rigidly fixed, which is similar to the characteristics of fracture repair in relatively stability [23], the initial bone growth at both ends of the prosthesis may be related to the stress stimulation of bone and the biological environment provided by membrane induction. All of the above factors may facilitate bone integration at the prosthesis-bone interface without bone graft, which can avoid the requirement and the complications of bone graft. From our cases observation, we found that osteogenesis occurred at the contact end of the bone and prosthesis, callus gradually increased, and crawled along the periphery of the prosthesis and around the contact surface of the prosthesis. Radiographies showed no complications such as implant loosening or subsidence, and our animal experiments [16] showed that osteogenesis was fully inserted into the prosthesis and crawled from both ends into the pore of the prosthesis.

There are limitations inherent in this approach. First of all, the number of patients is still small, more cases are needed to find the healing rules between prosthesis and bone. Secondly, we are not sure whether the intramedullary nail can be removed to observe the settlement between the prosthesis and bone. Thirdly, Titanium printed prostheses may have limited osteo-inductive ability, filling the porous structure of the prostheses with inducible drugs and factors is likely to achieve the effect of controlling infection and accelerating bone healing. Finally, these devices are not biodegradable. Further research is still needed.

\section{Conclusion}

3D printing technology could help to reconstruct local anatomy rapid, restore local biomechanical strength to allow early weight-bearing and exercise, which is beneficial for social function recovery. Biomechanical stimulation combined with biological induction may stimulate osteogenesis, achieve bone growth and stability at the bone/prosthesis interface, and greatly reduce the need for bone graft. Form our study, meticulous custom designed 3D printing technique of micro-porous prosthesis combined membrane induction and intramedullary fixation may be a cost-effective strategy to treat large metaphyseal femoral irregular-shaped bone defect without bone graft. This may be an alternative treatment for metaphyseal segmental bone defects, especially for cases of massive juxta-articular bone loss.

\section{Abbreviations}


PMMA: Polymethyl Methacrylate; CT: computed tomography; EMR: electronic medical record; IM: intramedullary nail; ROM: range of motion; AP: anterior-posterior; L: length; T: time; DRB: distal residual bone; PWB: partial weight bearing; FWB: total weight bearing

\section{Declarations}

\section{Authors' contributions:}

GJH and BCL were involved in designing the prosthesis, acquisition of data, drafting the manuscript. YT, ZJL and FZ designed the study, revised the manuscript critically and gave some important suggestions; $Y T, H Q J, Z S Z, Y G$ and YL made substantial contributions to conception and design and performed the surgeries; ZWY completed the follow-up and collected the datas. All authors read and approve the final manuscript.

\section{Author details:}

1 Department of Orthopaedic Surgery, Peking University Third Hospital, No 49, North Garden Rd, HaiDian District, Beijing 100191, China

2 Engineering Research Center of Bone and Joint Precision Medicine, Ministry of Education, Peking University Third Hospital, No 49, North Garden Rd, HaiDian District, Beijing 100191, China

\section{Competing interests:}

The authors declared that they have no competing interests in the authorship and publication of this contribution.

\section{Availability of data and materials:}

The datasets used and/or analysed during the current study are available from the corresponding author on reasonable request.

\section{Consent for publication:}

The consents for publication including any individual details, images with our institutional consent forms.

Acknowledgements: The authors would like to thank Changdong Qiu for the help of design the implant, who is a senior orthopaedic engineering professional for Shandong Weigao Orthopaedic Device Company Limited, the manufacturer of the implants described in the paper. Also like to Thank Xiaoyan $\mathrm{Niu}$, the secretary, for a lot of image preparation and data transmission work, which ensured the smooth progress of the project.

Ethics approval and consent to participate: 
This study was approval by Peking University Third Hospital Human Research Ethical Committees with the number M2018174. All patients were informed preoperatively regarding the surgical purpose, management protocol, possible complications and recovery period. And informed consent was obtained from all participants.

\section{Funding:}

This work was supported by Beijing Municipal Science \& Technology Commission (Project Z181100001718195).

\section{References}

1. Reichert JC, Saifzadeh S, Wullschleger ME, Epari DR, Schütz MA, Duda GN, Schell H, Van Griensven $M$, Redl H, Hutmacher DW. The challenge of establishing preclinical models for segmental bone defect research[J]. Biomaterials, 2009, 30(12):2149-2163.

2. Lindsey RW, Gugala Z, Milne E, Sun M, Gannon FH, Latta LL. The efficacy of cylindrical titanium mesh cage for the reconstruction of a critical-size canine segmental femoral diaphyseal defect[J]. Journal of Orthopaedic Research, 2006, 24(7):1438-1453.

3. Mauffrey C, Barlow BT, Smith W. Management of Segmental Bone Defects[J]. Journal of the American Academy of Orthopaedic Surgeons, 2015, 23(3):143-153.

4. Attias N, Lehman RE, Bodell LS, Lindsey RW. Surgical Management of a Long Segmental Defect of the Humerus Using a Cylindrical Titanium Mesh Cage and Plates[J]. Journal of Orthopaedic Trauma, 2005, 19(3):211-216.

5. Grant CA, Izatt MT, Labrom RD, Askin GN, Glatt V. Use of 3D Printing in Complex Spinal Surgery[J]. Techniques in Orthopaedics, 31(3):172-180.

6. Tetsworth KD, Mettyas T. Overview of Emerging Technology in Orthopedic Surgery[J]. Techniques in Orthopaedics, 31(3):143-152.

7. Song EK, Seon JK, Park SJ, Seo HY. Navigated Open Wedge High Tibial Osteotomy[J]. Sports Medicine and Arthroscopy Review, 2008, 16(2):84-90.

8. Jun Y, Choi K. Design of patient-specific hip implants based on the 3D geometry of the human femur[J]. Advances in Engineering Software, 2010, 41(4):537-547.

9. Buford WL, Turnbow BJ, Gugala Z, Lindsey RW. Three-Dimensional Computed Tomography-Based Modeling of Sagittal Cadaveric Femoral Bowing and Implications for Intramedullary Nailing[J]. Journal of Orthopaedic Trauma, 2014, 28(1):10-16.

10. Lu S, Zhang YZ, Wang Z, Shi JH, Chen YB, Xu XM, Xu YQ. Accuracy and efficacy of thoracic pedicle screws in scoliosis with patient-specific drill template[J]. Medical \& Biological Engineering \& Computing, 2012, 50(7):751-758.

11. Kataoka T, Oka K, Miyake J, Omori S, Tanaka H, Murase T. 3-Dimensional Prebent Plate Fixation in Corrective Osteotomy of Malunited Upper Extremity Fractures Using a Real-Sized Plastic Bone Model 
Prepared by Preoperative Computer Simulation[J]. The Journal of Hand Surgery, 2013, 38(5):909919.

12. Gouin F, Paul L, Odri GA, Cartiaux O. Computer-Assisted Planning and Patient-Specific Instruments for Bone Tumor Resection within the Pelvis: A Series of 11 Patients[J]. Sarcoma, 2014, 2014:1-9.

13. Cobos JA, Lindsey RW, Gugala Z. The Cylindrical Titanium Mesh Cage for Treatment of a Long Bone Segmental Defect: Description of a New Technique and Report of Two Cases[J]. Journal of Orthopaedic Trauma, 2000, 14(1):54-9.

14. Tetsworth K, Block S, Glatt V. Putting 3D modelling and 3D printing into practice: virtual surgery and preoperative planning to reconstruct complex post-traumatic skeletal deformities and defects[J]. SICOT-J, 2017, 3:16.

15. Xu N, Wei F, Liu XG, Jiang L, Cai H, Li ZH, Yu M, Wu FL, Liu ZJ. Reconstruction of the Upper Cervical Spine Using a Personalized 3D-Printed Vertebral Body in an Adolescent With Ewing Sarcoma[J]. Spine, 2015, 41(1).

16. Yang J, Cai H, Lv J, Zhang K, Leng HJ, Sun CG, Wang ZG, Liu ZJ. In Vivo Study of a Self-Stabilizing Artificial Vertebral Body Fabricated by Electron Beam Melting[J]. Spine, 2014, 39(8):E486-E492.

17. Hamid KS, Parekh SG, Adams SB. Salvage of Severe Foot and Ankle Trauma With a 3D Printed Scaffold[J]. Foot \& Ankle International, 2016, 37(4).

18. Cao JM, Zhou YJ, Zhu QH. Elective Ilizarov Bone Transport Technique in the Treatment of Infected Tibial Bone Defect[J]. Journal of Practical Orthopaedics, 2016, 22(11).

19. Lacroix D, Prendergast PJ, Li G, Marsh D. Biomechanical model to simulate tissue differentiation and bone regeneration: Application to fracture healing[J]. Medical \& Biological Engineering \& Computing, 2002, 40(1):14-21.

20. Xiu P, Jia Z, Lv J, Yin C, Cheng Y, Zhang K, Song C, Leng H, Zheng Y, Cai H, Liu Z. Tailored surface treatment of 3D printed porous Ti6Al4V by micro-arc oxidation for enhanced osseointegration via optimized bone in-growth patterns and interlocked bone/implant interface[J]. ACS Applied Materials \& Interfaces, 2016:acsami.6b05893.

21. Liu H, Hu G, Shang P, Shen Y, Nie P, Peng L, Xu H. Histological characteristics of induced membranes in subcutaneous, intramuscular sites and bone defect[J]. Orthopaedics \& Traumatology: Surgery \& Research, 2013, 99(8):959-964.

22. Gruber HE, Gettys FK, Montijo HE, Starman JS, Bayoumi E, Nelson KJ, Hoelscher GL, Ramp WK, Zinchenko N, Ingram JA, Bosse MJ, Kellam JF. Genomewide Molecular and Biologic Characterization of Biomembrane Formation Adjacent to a Methacrylate Spacer in the Rat Femoral Segmental Defect Model[J]. Journal of Orthopaedic Trauma, 2013, 27(5):290-297.

23. Perren SM, Rahn BA. Biomechanics of fracture healing[J]. Canadian Journal of Surgery Journal Canadien De Chirurgie, 1980, 23(3):228-32.

\section{Figures}




\section{Figure 1}

A 61ys old male had open fracture of his bilateral femur caused by motor vehicle accident. A: debridement and ORIF have been done. Unfortunately, there was an infectious bone defect. B: debridement of all the infected bone, PMMA spacer and external fixation was done. C: the affected limb is about $3 \mathrm{~cm}$ shorter than the contra-lateral limb. D,E: postoperative X-ray showed that the prosthesis matched well with the defect area. F: the length of affected limbs was nearly reconstructed. G,H: 1 month post-op X-ray showed good position of prosthesis and internal fixation, callus formation at prosthesisbone interface (red arrow). I,J: 12months post-op X-ray demonstrated good osseous integration of the prosthesis-bone interface (yellow arrow). No signs of subsidence, implant collapse, or loss of correction. $\mathrm{K}, \mathrm{L}$ : function of the knee.

\section{Figure 2}

A,B: A 42-year-old female have open fracture of her right femur caused by motor vehicle accident 3years ago. C,D: debridement and ORIF have been done. E,F,G: X-ray and CT showed nonunion and large segmental bone defect. Clinical and laboratory examinations exclude infection. $\mathrm{H}$ : debridement of the non-viable bone and interposed fibrous tissue, PMMA spacer remained in the defect for 8 weeks, during which time the implant was designed and manufactured. I,J: postoperative X-ray showed that the prosthesis matched well with the defect area. K,L: 4 months post-op X-ray showed good position of implant and internal fixation, callus formation(red arrow). M,N: 12-month post-op X-ray demonstrated good osseous integration of the prosthesis-bone interface. No early signs of subsidence, implant collapse. O,P: hip and knee function were good.

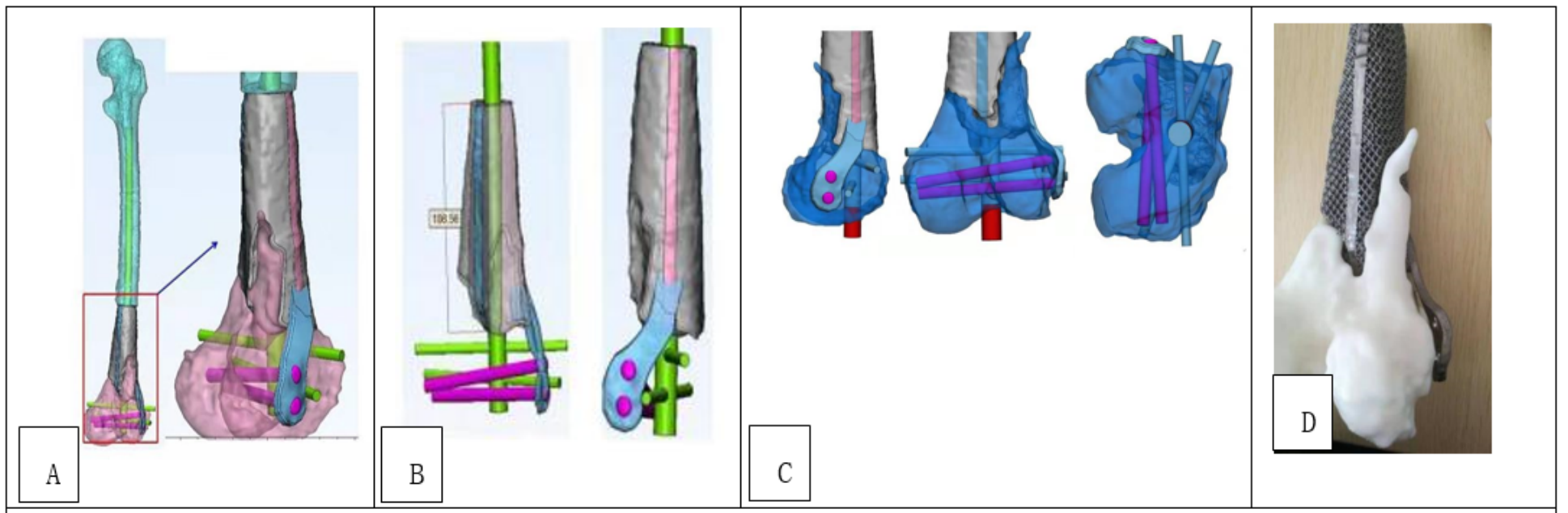

\section{Figure 3}

$A, B$ : the prosthesis should be configured to mimic the size and shape of the original bone(equal radius of curvature and modulus of elasticity). If the distal end of the prosthesis is too close to the knee joint or the 
distal residual bone quality is poor, a lateral plate was added to ensure strength. C: $6.5 \mathrm{~mm}$ cancellous bone screw should be used for fixation, which should avoid interference with intramedullary nail locking screw. D: the prosthesis was also validated on the model. 